\title{
Integrating Bus Holding Control Strategies and Schedule Recovery: Simulation-Based Comparison and Recommendation
}

\author{
Weitiao Wu $\mathbb{D}^{1},{ }^{1}$ Ronghui Liu $\mathbb{D}^{2},{ }^{2}$ and Wenzhou Jin ${ }^{1}$ \\ ${ }^{1}$ South China University of Technology, Wushan Road, Tianhe District, Guangzhou 510641, China \\ ${ }^{2}$ University of Leeds, Institute for Transport Studies, Leeds LS2 9JT, UK \\ Correspondence should be addressed to Weitiao Wu; ctwtwu@scut.edu.cn
}

Received 7 April 2018; Accepted 21 June 2018; Published 17 July 2018

Academic Editor: Ondrej Stopka

Copyright (c) 2018 Weitiao Wu et al. This is an open access article distributed under the Creative Commons Attribution License, which permits unrestricted use, distribution, and reproduction in any medium, provided the original work is properly cited.

\begin{abstract}
In the absence of control strategies, headway fluctuation and bus bunching are commonly observed in transit operation due to the stochastic attributes such as travel time and passenger demand. Existing research on real-time control largely focused on developing operational tactics to maintain bus arrival regularity at stops without fully considering the effect of schedule recovery. This paper investigates the effect of bus driver behavior on bus holding control strategies and more specifically their effort in catching up with schedule in case of delay, i.e., schedule recovery. To this end, this paper first proposes a bus propagation model with capacity constraint to simulate the evolution of bus trajectories along a fixed route. It proceeds to explicitly incorporate both holding control actions and schedule recovery effect into the bus propagation model. Using simulation for a high-frequency bus line in Guangzhou, China, schedule- $(\mathrm{SH})$ and headway-based holding $(\mathrm{HH})$ control strategies are compared under various operational settings in the context of schedule recovery. These comparisons show that SH performs better under certain conditions, and SH generally benefits more from schedule recovery than $\mathrm{HH}$. These results provide insights into the bus stop layout design and implementation of holding methods in the context of cruising guidance.
\end{abstract}

\section{Introduction}

Service reliability of public transport system is of great importance to passengers. Studies have shown that passengers value travel time reliability four times higher than they do to average travel time [1]. In the uncontrolled bus systems, buses are likely to bunch in the presence of stochastic travel time and demand, which is commonly observed in the peak hours. Bus bunching occurs when a number of buses arrive at a stop within an interval that is shorter than schedule headway or even together. Such phenomenon is undesirable for both passengers and the operator since it leads to unpredictable bus arrival times and additional waiting time at stops, which discourages passengers from choosing public transport.

A variety of operational tactics have been proposed to improve bus system performance in the literature, while holding control is the most commonly used [2]. The holding controlling approaches can be classified into three groups, including schedule-based control, headway-based control, and optimization-based control. They works by injecting slack time into the schedule at designated stops, in which the slack time refers to the amount of time that a task in a schedule can be delayed without causing a delay to subsequent tasks. The first two methods are triggered by bus arrival time and headway deviations, respectively, while the other one optimizes holding times through formulating a mathematical programming problem based on cost or time minimization. In this sense, the slack times are predetermined static settings for schedule-based control, whereas they are determined in real-time for headway- and optimization-based control. Osuna and Newell [3] investigated the holding problem at a single point for a cyclical route, in an attempt to minimizing the overall passenger waiting time. Hickman [4] developed an analytical model to determine the optimal holding time at a control stop along a bus route considering stochastic running times. Eberlein et al. [5] formulated the holding problem as a deterministic quadratic program with the availability of realtime information. Zhao et al. [6] studied the determination 
of optimal slack time under schedule-based control with the aim of minimizing the expected waiting time of passengers.

Daganzo [7] proposed a headway-based control scheme, where the holding times are dynamically determined by the information of the forward headway. It was found that the proposed method could achieve a faster speed and thus lower travel time compared to the schedule-based approach. Later, Xuan et al. [8] developed a family of control strategies by combining both the forward and backward headway information. Results show that such scheme can considerably reduce slack times and enhance headway regularity. This work was extended by Argote-Cabanero et al. [9] to be generalized to evaluate a bus corridor with multiple bus lines. Daganzo and Pilachowski [10] proposed an adaptive control scheme based on vehicle-to-vehicle cooperation, in which the bus cruising speed was adjusted in a real-time manner with the information of expected demand and vehicle spacing. They reported that the scheme yields regular headways with faster travel than the earlier control strategies. Delgado [11] developed a holding control policy in combination with boarding limit in an attempt to minimizing total delays on the transit corridor. Hernandez et al. [12] presented an extended model considering multiple bus line services. Recently, Sánchez-Martínez et al. [13] formulated a holding control model with dynamic running times and demand.

The variability in travel time is one of the central sources triggering bunching. Some efforts were taken to tackle the effects of exogenous variables on the travel time variability by employing machine learning models and predictive analytics that are able to explain such variability to learn about the behavior of a given fleet of vehicles. In this way, proactive control approaches could be generated that are able to prepare the system for delays or surges in demand before they reach a critical level. For example, Yu and Yang [14] used a support vector machine to predict the arriving status in the implementation of holding control. Moreira-Matias et al. [15] integrated the bus bunching prediction model into a real-time framework to mitigate bus bunching, of which the prediction output is used to select and employ corrective actions (holding and stop skipping). Nair et al. [16] presented real-time predictive analytics for bus bunching by using the real data in Miami-Dade Transit. Recently, Andres and Nair [17] presented a predictive-control framework to reduce bus bunching, which involves hierarchically related components including headway prediction and dynamic holding control. There are also literatures that investigate the exogenous factors affecting bus bunching, such as passenger arrival patterns (Fonzone et al. [18]) and the presence of common line (Schmöcker et al. [19]). Holding strategies have also been used for transfer synchronization. As direct and transfer demand are the mainstreams of transit networks [20], such problem is usually formulated as minimizing passenger waiting time or cost accounting for these types of passengers. Recently, Wu et al. [21] incorporated schedulebased holding control with a predefined time window into the bus schedule coordination design.

With the advances in connected vehicle technology and roadside detectors development such as time control points, the information of schedule deviations can be readily collected and informed to bus drivers. This provides new opportunities for transit operators with real-time schedule adherence status. Due to the travel time variability, both early and late arrivals can occur when compared to the reference timetable at the designated time control stops. One of the operational goals of a transit agency is to maintain buses on schedule. In reality, well-experienced bus drivers constantly adjust their speeds to keep their buses on schedule [22-25]. Figure 1 shows a potential way of realizing this goal using cruising guidance with colored bars that move up and down, such that drivers are able to vary their average speed to improve the schedule adherence. According to an empirical study conducted by a transit agency in the northeastern United States, schedule recovery effort can be observed on at least half of the segments on a bus route [22]. Recently, Liu et al. [26] proposed an inter-vehicle communication scheme to achieve a planned direct transfer. Two operational tactics were employed by using real-time information: speed control and holding at transfer point, of which speed control resembles schedule recovery behavior.

Schedule-based holding control are often employed when the bus arrives earlier than the scheduled arrival time. When a bus arrival is behind schedule, schedule recovery tactics could be deployed to support/guide the driver to catch up with the schedule at the next time control point. Unlike holding control that keeps buses at stops, the schedule recovery emphasizes speed adjustment between stops. This inter-stop control action can be utilized as complementary to holding control. Thus there may exist interactions between schedule recovery and holding control. The performance of bus scheduling is closely related to the dynamic motion of buses including driving behavior described by speed and acceleration. Therefore, schedule recovery behavior should be taken into consideration in the execution of holding control strategy. However, most of the existing literatures on holding control have focused on developing and evaluating the effectiveness of different action rules, largely neglecting the inherent effect of such schedule recovery driving behavior.

On the other hand, there is a set or "library" of feasible operational tactics to be used by transit operators. Among them, speed adjustment and vehicle holding are usually employed as combinatorial strategies. Speed adjustment, when applied in bus delay scenarios, resembles schedule recovery. For example, Nesheli et al. [27] used a combination of speed change control and headway-based holding control to reduce bus bunching. On a similar combination of strategies, Milla et al. [28] integrated holding and stop-skipping control based on fuzzy rules to minimize users' travel time. While headway-based and schedule-based control are two distinctive and most commonly implemented holding control methods, no performance comparison was made between them in the combinational design of operational tactics in the literature. Understanding the combined effect could help to design proper combinational tactics in response to varying traffic conditions.

The major focus of this paper is to compare the combined effect of schedule recovery and two different holding control approaches, so as to evaluate how cruising guidance technology and the resulting schedule recovery behavior affects 

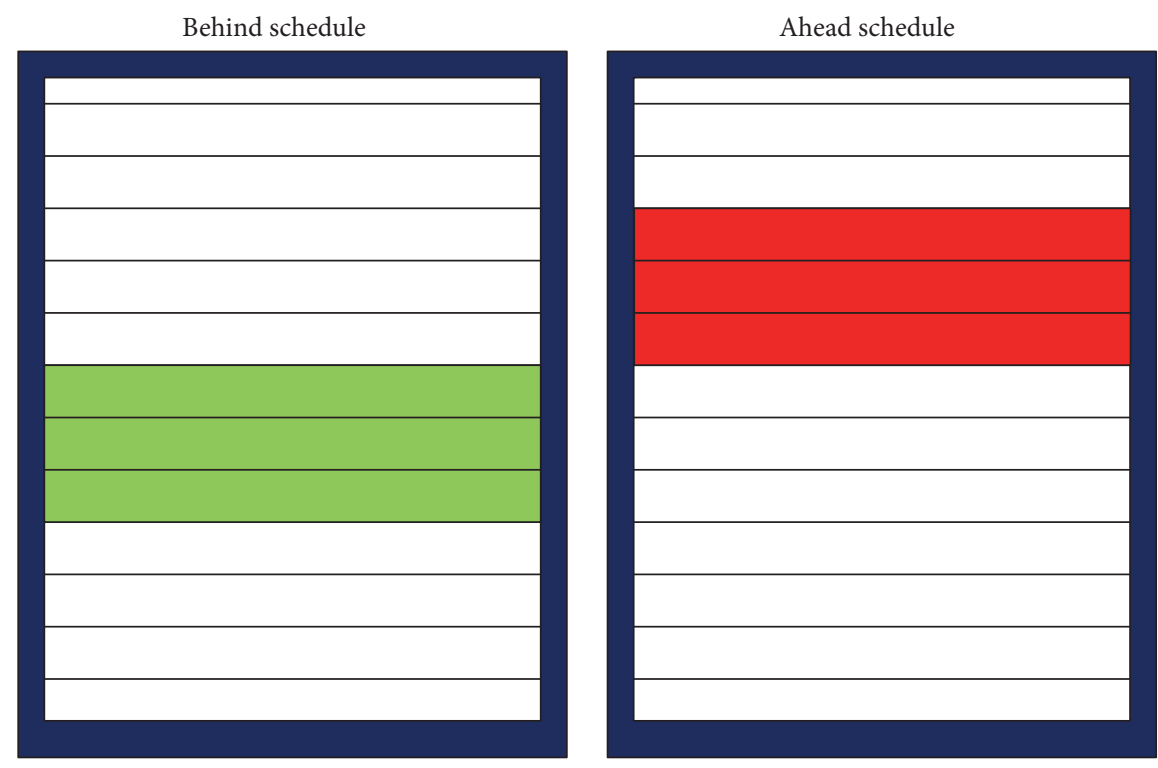

FIGURE 1: Cruising guidance on-board device surface (adapted from [9] ).

the bus holding control strategies. This comparison should allow us to identify the scenarios for which the different holding control methods present advantages and highlight their respective strengths for further implementation in the context of cruising guidance technology. We have made an effort in this document to present both approaches with a common nomenclature. Our findings show that schedule recovery plays an important role in the design of bus holding control and that, for specific indicators, the optimal holding strategy transition will occur with certain level of schedule recovery effort and under certain conditions. We thus suggest the bus operators should select the most appropriate operation strategy that suits their operating conditions, which provides managerial insights into bus operational control. To the best of our knowledge, this is the first time that holding control strategies are compared in the context of schedule recovery.

The remainder of this paper is organized as follows. In Section 2, simulation frameworks are developed. In Section 3, performance measures are introduced to evaluate the performance of bus service. In Section 4, model experiments are performed and their practical implications are provided. Finally, the conclusions and future research directions are given in Section 5.

\section{Modelling Approach}

This paper is designed to investigate the effects of schedule recovery on the performance of holding control strategies, more specifically on the schedule-based and headway-based holding control. The main objectives are threefold: (a) develop an enhanced bus propagation and holding control models, which explicitly takes the schedule deviation and driving behavior into account; (b) compare the performance of the two holding control strategies under various operational settings in the context of schedule recovery; and (c) discuss the implementation issue for holding control with schedule recovery.

2.1. Assumption and Notations. Without loss of generality, the following assumptions are made:

(A1) Passenger arrival pattern relates directly to bus headways ([29]). In this paper passenger arrivals at bus stops is assumed to follow Poisson distributions. This assumption is reasonable on high-frequency routes, as has already been validated and commonly used by many researchers (e.g., $[14,30,31])$

(A2) The boarding process and the variability in link travel time are attributing factors to bus bunching.

(A3) Bus overtaking maneuvers are prohibited. This is reasonable since overtaking rarely occurs under the combined effect of holding and schedule recovery. This is also a common simplification in the literature (e.g., [13]). When overtaking is allowed, the bus order may change from stop to stop. Thus allowing overtaking requires structural changes to the model which has been left for another work.

(A4) Over the study-time horizon, passenger demand is assumed to be stochastic governing Poisson distribution, while bus running times vary at results from stochastic phenomena in the network.

2.2. Bus Propagation Model with Capacity Constraint. A bus motion model is comprised of three components: departures of buses, dwell times at stops, and link travels times. The arrival time of bus $i$ at stop $j$ is the departure time from stop $j-1$ plus the random link travel time between stop $j-1$ and j:

$$
a_{i, j}=d_{i, j-1}+t_{i, j-1}
$$


The uncontrolled bus departure time is determined by its arrival time and dwell time:

$$
d_{i, j}=a_{i, j}+D_{i, j}
$$

Following Liu et al. [32], the headway between bus $i$ and the preceding one is assumed to be the gap between bus $i-1$ leaving stop $j$ and bus $i$ arriving stop $j$.

$$
h_{i, j}=a_{i, j}-\bar{d}_{i-1, j}
$$

where $\bar{d}_{i-1, j}$ stands for the previous departure time from the control point, which is equal to $d_{i-1, j}$ plus the corresponding holding time. $d_{i-1, j}$ is effectively equal to $\bar{d}_{i-1, j}$ without holding control.

Passenger arrival is assumed to be stochastic governing Poisson process, with the mean arrival flow equals to the product of the mean passenger arrival rate $q_{j}$ and the headway $h_{i, j}$ of the bus with its leader. Therefore, the boarding demand for bus $i$ at stop $j$ is

$$
B_{i, j}=P\left(q_{j} h_{i, j}\right)+l_{i-1, j}
$$

The alighting demand is drawn from a binomial distribution, which is related to the bus load $L_{i, j}$ before arriving at stop $j$ and its alighting percentage $\rho_{j}$.

$$
A_{i, j}=B i\left(L_{i, j-1}, \rho_{j}\right)
$$

With vehicle capacity constraint, the actual number of boarding passengers is either the boarding demand or the remaining capacity, then we have

$$
\begin{aligned}
\bar{B}_{i, j} & =\min \left\{B_{i, j}, C-L_{i, j-1}+A_{i, j}\right\} \\
& =\min \left\{B_{i, j}, C-L_{i, j-1}\left(1-\rho_{j}\right)\right\}
\end{aligned}
$$

We assume that waiting passengers are loaded in a random fashion, which is appropriate when passengers mingle on waiting platforms. It is further assumed that each available space is equally likely to favoured by the waiting passengers, thus the boarding probability is the actual number of boarding passengers to the boarding demand, i.e., $\bar{B}_{i, j} / B_{i, j}$. Evidently, when the number of passengers who want to board exceed the remaining capacity, the probability is less than 1 ; otherwise, the probability is equal to 1 . Therefore, the actual number of arriving passengers who are able to board is

$$
\bar{B}_{i, j}^{\prime}=\frac{\bar{B}_{i, j}}{B_{i, j}} P\left(q_{j} h_{i, j}\right)
$$

Equations (6) and (7) are used to calculate the average waiting time; see Section 3.2.

Therefore, the number of leftover passengers is the difference between total boarding demand and the actual number of boarding passengers.

$$
l_{i, j}=B_{i, j}-\bar{B}_{i, j}
$$

The number of on-board passengers in bus $i$ when it departs from stop $j$ is related to the load before arriving the current stop and passenger flow at current stop.

$$
L_{i, j}=L_{i, j-1}+\bar{B}_{i, j}-A_{i, j}
$$

When the vehicle is not crowded $\left(L_{i, j} / C \leq \varphi\right.$, where $0<$ $\varphi<1$ is a constant), passenger boarding and alighting take place simultaneously in a front-on rear-off policy. Thus the bus dwell time at the stop is estimated as the maximum time between the boarding and alighting time, plus the open and close door time.

$$
D_{i, j}=\max \left\{b \bar{B}_{i, j}, \alpha A_{i, j}\right\}+\tau
$$

where $\varphi$ is a threshold of in-vehicle crowding degree. $\tau$ is the open and close door time. $b$ and $\alpha$ represent the average boarding time and alighting time for passengers.

Note that the link travel time extracted from GPS data includes the acceleration and deceleration time.

If the vehicle is crowded, i.e., $\varphi<L_{i, j} / C \leq 1$, the passengers would need more time to board and alight, and the dwell time is

$$
D_{i, j}=\gamma \max \left\{b \bar{B}_{i, j}, \alpha A_{i, j}\right\}+\tau
$$

where $\gamma$ is the crowding coefficient, $\gamma>1$.

2.3. Bus Holding Control with Schedule Recovery. When realtime holding control is in place, the bus departure time may be modified according to the control policy. This inherently has an effect on the boarding and alighting process. In this paper, two typical control methods are investigated: the schedule-based and headway-based holding control strategies. Schedule recovery is only triggered by schedule deviation independent of control strategies. Such time deviation can be readily informed to drivers. The driver adjustment is related to the schedule adherence status of the bus. In what follows, the corresponding schedule deviations are identified and the effect of schedule recovery are incorporated.

2.3.1. Schedule-Based Holding Control (SH). Under SH, buses either depart on schedule or immediately after serving passengers if they arrive late at the time point [2]. Therefore, the scheduled departure time takes the following piecewise function:

$$
d_{i, j}= \begin{cases}s_{i, j}, & a_{i, j}<s_{i, j}-D_{i, j} \\ a_{i, j}+D_{i, j}, & a_{i, j} \geq s_{i, j}-D_{i, j}\end{cases}
$$

where $s_{i, j}-D_{i, j}$ is the critical arrival time after which the bus has to depart later than the scheduled departure time $s_{i, j}$.

The scheduled departure time at a designated stop $s_{i, j}$ can be calculated as the scheduled departure time from the previous stop $s_{i, j-1}$ plus the scheduled link travel time. The dwell time is included in the scheduled link travel time. The reliability of bus operation under $\mathrm{SH}$ depends on the scheduled link travel time. The scheduled link travel 
time could be set as the average link travel time multiplied by a safety factor (we term it slack ratio) to provide time redundancy and thereby absorbs travel time randomness.

When $a_{i, j}<s_{i, j}-D_{i, j}$, the early arriving bus will be held until time $s_{i, j}$. The schedule deviation, and therefore schedule recovery, arises when the bus arrives at a designated stop later than the critical arrival time, i.e., when $a_{i, j} \geq s_{i, j}-D_{i, j}$, then the delay experienced by bus $i$ at stop $j$ is $d_{i, j}-s_{i, j}$. Similar to Chen et al. [22] and Yan et al. [25], the driver's adjustment between the current time point and the preceding time point is assumed to be proportional to the schedule deviation at the current point. Therefore, the driver adjustment can be estimated as $\beta_{i, j}\left(d_{i, j}-s_{i, j}\right)$. As a result, by modifying (1), the arrival time with schedule recovery is

$$
a_{i, j+1}=d_{i, j}+t_{i, j}-\beta_{i, j}\left(d_{i, j}-s_{i, j}\right)
$$

where $\beta_{i, j}$ represents the adjustment factor between stop $j$ and $j+1$ for bus $i$. In practice, this adjustment parameter can be estimated from historical trip information and is a stochastic variable following a specific distribution will be discussed in Section 2.3.3.

2.3.2. Headway-Based Holding Control (HH). HH approach is usually triggered by headway deviation. In line with $\mathrm{SH}$ control, schedule recovery in $\mathrm{HH}$ works when arrival delay arises. In this study, we use a heuristic $\mathrm{HH}$ similar to that proposed by Sánchez-Martínez et al. [13]. The rational is that hold bus $i$ at control point $j$ to ensure preceding headways are never less than a prescribed design headway. In order to attain the desired headway, when the current headway is smaller than the desired headway, the vehicle should be held at the stop; otherwise, the schedule recovery should be employed. The recovery time is based on the headway deviation. The scenarios are specified as follows.

Scenario $I$. When the headway is shorter than the design headway, i.e., $h_{i, j} \leq H$, hold bus $i$ at stop $j$ for time $H-h_{i, j}$, thus the arrival at the next stop is simply expressed as

$$
a_{i, j+1}=\bar{d}_{i, j}+t_{i, j}
$$

Scenario II. When the headway is larger than the design headway, i.e., $h_{i, j}>H$, the bus should depart immediately and schedule recovery starts, and the arrival at the next stop is

$$
a_{i, j+1}=d_{i, j}+t_{i, j}-\beta_{i, j}\left(h_{i, j}-H\right)
$$

where $h_{i, j}-H$ represents the schedule deviation under headway-based holding control, which can be informed to the bus driver for schedule recovery instruction immediately when bus $i$ completes serving passengers at stop $j$.

2.3.3. Calibration of Schedule Recovery Factor. As discussed, bus drivers tend to actively pursue schedule recovery if the bus is delayed. Naturally, driver's behavior is highly dependent on the his/her experience, which may vary considerably from scenario to scenario, fleet to fleet, or even from vehicle to vehicle. Therefore, it is reasonable to assume that the schedule recovery factor follows a specific distribution. Based on automatic vehicle location (AVL), automatic passenger counting (APC) data, and the timetable, the delay at stops could be calculated and then correlated with the travel time deviation on the next leg of the journey to the next downstream stop. Consequently, using the historical trip data, one can get the distribution of the adjustment factor $\beta_{i, j}$ along the route.

According to the empirical study by Chen et al. [22], the average adjustment factors vary within a range between -0.5 and 0.5 on most segments. Since the early arrival will be compensated by holding control, here we assume the adjustment factor is nonnegative; that is, bus drivers are always trying to recover the schedule deviation at the preceding time control point.

2.4. Solution Algorithms for Bus Trajectories Evolution. Instead of using simulation tools such as multiagent approach or discrete event-based simulation software (e.g., [33]), in this study we develop bus propagation models to simulate the evolution of bus motion and evaluate the bus holding methods. Unlike the disaggregated models in which system dynamics are explicitly simulated by individual travel behavior, in our simulation framework the passengers' activity is modelled in an aggregated way. The advantage of aggregate models is their greater computational efficiency, which facilitates repeated simulation.

With the above formulations, Algorithm 1 outlines the general simulation framework in which alighting process, capacity constraint, and leftover passengers are incorporated. The algorithm is made up of three components: calculating, respectively, the departures of buses, link travel times, and dwell times. We consider a unidirectional bus route where vehicles depart from one terminal to another. Although extension from one-way line to the general bus route with bidirectional traffic would be straightforward, the modelling a unidirectional route avoids considering traffic continuity at terminal stations and fleet size limitation problem as with the cyclic route where inbound and outbound headways are correlated. Since late or early arrivals at terminals can occur due to travel time variability [34], modelling cyclic route may also result in departure headway fluctuations from the terminals. Such effect is a special case of travel time variability. Let $M$ denote the fleet size of the modelled bus line and $N$ the number of bus stops on the corridor served by the bus line. To discourage bunching at the beginning of the simulation, headways are set deterministic and at a uniform headway; thereafter headways become stochastic. In order to make the system evolve to be chaotic enough for bus bunching to appear, the number of buses $M$ is set sufficiently large in each run of simulation. In this regard, one may consider the simulation of the first few buses in the system as a "warm-up" period.

In order to avoid bus overtaking phenomenon, the bus headway $h_{i, j}$ is required to be larger than a value. When the preceding bus is caught by the next incoming bus during the 


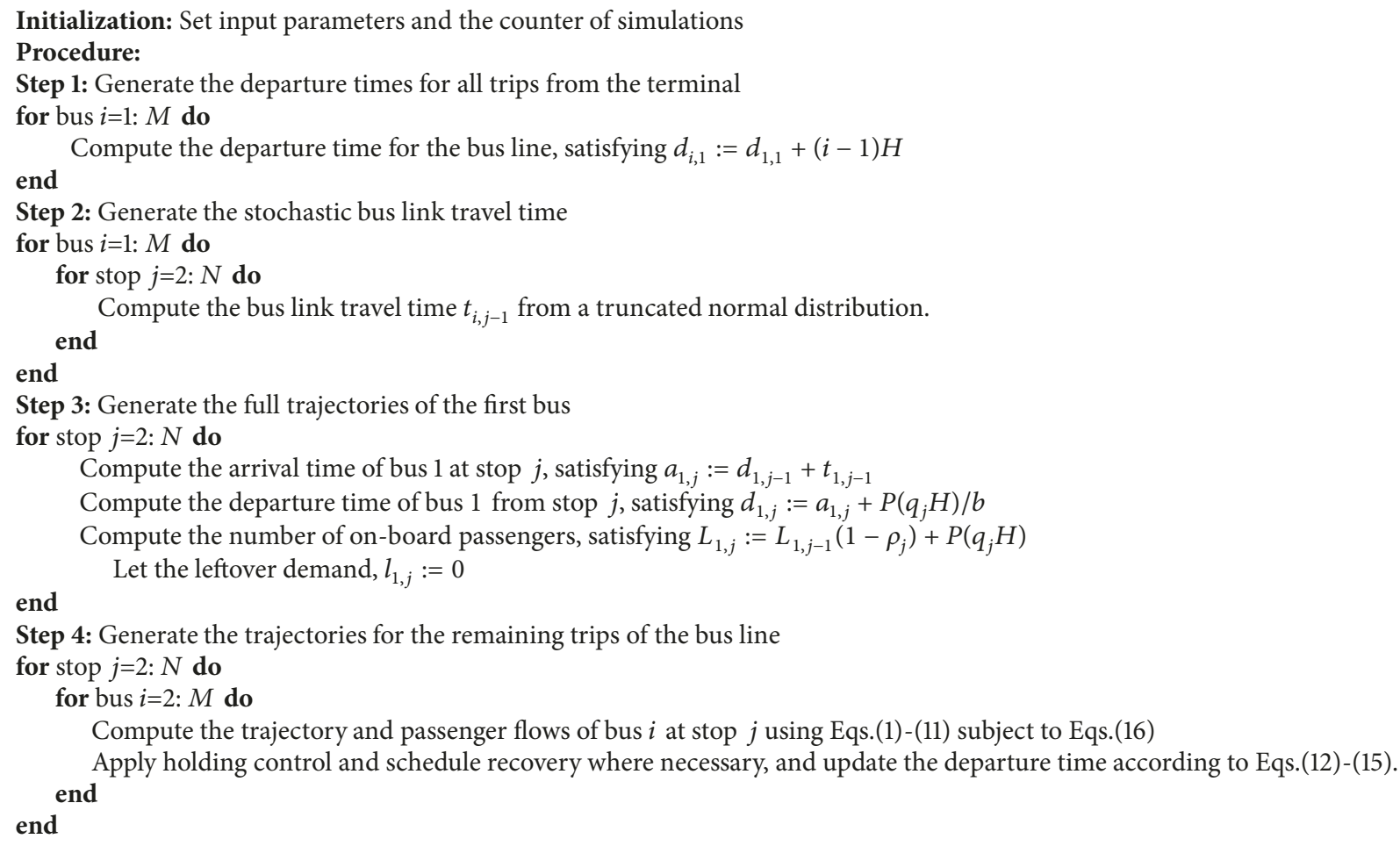

Algorithm 1: Bus trajectories evolution algorithm.

simulation, i.e., $h_{i, j}<0$, let the preceding bus restart after a delay time $\delta$, which we call it minimum safety interval, i.e.,

$$
a_{i, j}=\bar{d}_{i-1, j}+\delta
$$

\section{Performance Measure}

To evaluate the performance of different control strategies, we use a number of performance measures to take into account the views of different stakeholders: passengers and operator. The headway variability is the major concern of both passengers and operator, since uneven headway is the main cause of spatially uneven loads and thus bus bunching. The average waiting time reflects the level of service and appears to be uppermost for passengers. In addition, the operator may concern the travel time reliability, since it is crucial for schedule design and operation costs. A smoother and more robust operation and planning at terminals requires lower variability of travel time. Multiple simulation runs are conducted, from which we generate distributions of performance measures.

3.1. Headway Variability Coefficient (HVC). Similar to Turnquist and Bowman [35], Liu and Sinha [36], and Wu et al. [37], we use the headway variability coefficient (HVC) to measure the reliability of the observed headways, which is defined as the ratio of the standard deviation to the mean headway. This coefficient is the coefficient of variation as known in statistics and probabilities.
3.2. Average Waiting Time. As mentioned previously, in this study, the passengers' activity is modelled in an aggregated way; thus it would be difficult to obtain the waiting time of each individual passenger. The average passenger waiting time could be achieved by (6)-(8).

The waiting passengers at a station are divided into two groups: those who are able to board and those who are left behind due to capacity constraint. The former, of which the number is $\bar{B}_{i, j}^{\prime}$ (see (7)), arrives randomly during time window $\left[0, h_{i, j}\right]$; thus their expected waiting time can be approximated to be half of the headway $h_{i, j} / 2$ (SánchezMartínez et al. [13]; Salek and Machemehl [30]). The passengers who are left behind, $l_{i-1, j}$, have to wait for the next bus, and their additional waiting time is the entire headway $h_{i, j}$. Summing up these two groups we have the total waiting time expressed as $(1 / 2) \sum_{i} \sum_{j} \bar{B}_{i, j}^{\prime} h_{i, j}+\sum_{i} \sum_{j} l_{i-1, j} h_{i, j}$.

Dividing the total waiting time by the total number of boarding passengers $\bar{B}_{i, j}$ (see (6)), we have the average waiting time per passenger as follows:

$$
\begin{aligned}
\bar{w} & =\frac{(1 / 2) \sum_{i} \sum_{j} \bar{B}_{i, j}^{\prime} h_{i, j}+\sum_{i} \sum_{j} l_{i-1, j} h_{i, j}}{\sum_{i} \sum_{j} \bar{B}_{i, j}} \\
& =\frac{\sum_{i} \sum_{j}\left(\bar{B}_{i, j}^{\prime}+2 l_{i-1, j}\right) h_{i, j}}{2 \sum_{i} \sum_{j} \bar{B}_{i, j}}
\end{aligned}
$$

As shown in Section 2.2, the number of waiting passengers and the headways are interdependent. The expected 


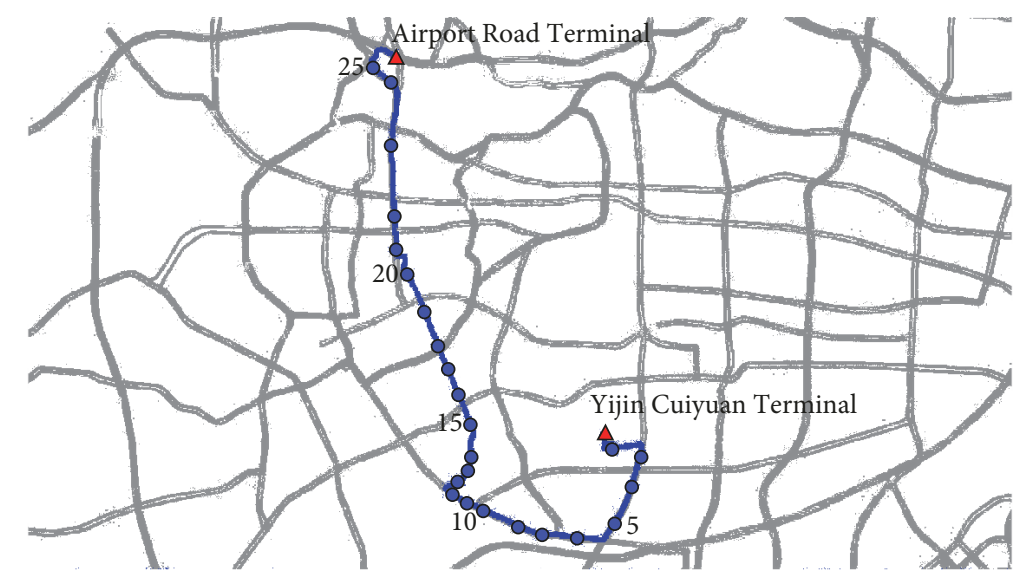

FIGURE 2: Bus route 87. The direction of the bus route discussed in this article is from the bottom to the up.

average waiting time $E(\bar{w})$ can be drawn from multiple simulation runs.

3.3. Average Travel Time. While bus holding control could improve the service regularity, it prolongs the terminal-toterminal running time for the vehicles. The average travel time for buses is an important operational performance measure for transit operators since it is related to the fleet size. In each run of simulation, an average travel time is derived as the arithmetic mean over all buses simulated. Then an expected average travel time, derived from multiple simulation runs, is used as the performance measure.

3.4. Load Variation. Headway variability and bus bunching lead to uneven load. Such spatially heterogeneous demand is one of the major sources triggering crowding effect since discomfort happens at high load factors. Therefore, a more balanced load factor across buses yields a more comfortable experience to users. In view of this, we introduce load variation to evaluate the performance of bus control strategies, which is defined as the standard deviation of all bus loads across all segments. Then the expected load variation can also be drawn from multiple simulation runs.

\section{Comparative Results on a Simulated Real-Life Route}

In this section, we compare the performance of two bus holding controls with and without schedule recovery (SR) effort: the simple schedule-based holding ( $\mathrm{SH}$ ) and headwaybased holding ( $\mathrm{HH}$ ) and SH with schedule recovery (SHSR) and $\mathrm{HH}$ with schedule recovery (HHSR).

These four control methods are applied to bus route 87 in the city of Guangzhou, China. The bus route (shown in Figure 2) has a total distance of $14.7 \mathrm{~km}$. It connects Yijin Cuiyuan Terminal and Airport Road Terminal in the city. The passenger demand and link travel time data are provided by a local bus company. We use the data during the morning peak hour (9:00-10:00 am) and in the direction from Yijin Cuiyuan
Terminal to Airport Road Terminal in the city. The scheduled headway of the route is taken as 8 minutes.

Following Liu et al. [32], we assume that passengers boarding at a station will evenly alight at the downstream stops; thus the stop-specific average alighting rate $\rho_{j}$ can be obtained from the boarding rate. Table 1 shows the empirical data on passenger arrival rate and the derived alighting distributions. The link travel time data are obtained from on-board GPS tracking devices, from which the mean and standard deviation of travel time between stops are calculated and listed in Table 2. At present, the bus route operates in an unscheduled and uncontrolled manner.

The minimum safety interval is set as $\delta=0.3 \mathrm{~min}$, the average boarding and alighting time are set as $\alpha=2 s$ and $b=4 s$, respectively, and vehicle capacity is set as $C$ $=100 \mathrm{pax} / \mathrm{veh}$. The open and close door time is taken as $\tau=4 s$. The threshold of the in-vehicle crowding degree $\varphi$ is set to be 0.8 . The crowding coefficient is set as $\gamma=$ 1.5 , and the number of buses $M$ is set as 20 . In order to highlight the relative effect of holding and schedule recovery, all intermediate stops are considered key time control stops. Buses are set to depart from the terminal on time in the base case. The link riding times are drawn from a truncated normal distribution with means and standard deviations as listed in Table 2. The simulation is run 1000 times.

The detailed output in a typical simulation includes vehicle trajectories, the vehicle load, and the number of leftover passengers. In what follows, we test the effectiveness of the simulation model under various operating settings. To represent differences in driver behavior and their impact on schedule recovery, in each simulation the schedule recovery factor $\beta_{i j}$ is randomly generated for each segment following a uniform distribution.

4.1. Slack Ratio for Schedule-Based Holding Control. The slack time is a crucial predetermined setting for $\mathrm{SH}$ control. To investigate, we define slack ratio as the multiplier of average link travel times, and we test the system performance for a range of slack ratios: $\{0.6,0.7,0.8,0.9,1,1.1,1.2,1.3,1.4,1.5\}$. 
TABLE 1: Observed passenger arrival flow and derived alighting proportion on 87 Route.

\begin{tabular}{lccccccccccccc}
\hline stop & 1 & 2 & 3 & 4 & 5 & 6 & 7 & 8 & 9 & 10 & 11 & 12 & 13 \\
\hline Arrival rate (pax/min) & 0.34 & 0.22 & 0.17 & 0.23 & 0.25 & 0.27 & 0.45 & 0.91 & 0.64 & 0.99 & 0.56 & 0.74 & 0.25 \\
Alighting Proportion (\%) & 0 & 4.2 & 4.3 & 4.5 & 4.8 & 5 & 5.3 & 5.6 & 5.9 & 6.3 & 6.7 & 7.1 & 7.7 \\
Stop & 14 & 15 & 16 & 17 & 18 & 19 & 20 & 21 & 22 & 23 & 24 & 25 & 0.14 \\
Arrival rate (pax/min) & 0.79 & 0.26 & 0.38 & 0.35 & 0.27 & 0.29 & 0.30 & 0.11 & 0.14 & 0.08 & 0.06 & 0.03 & \\
AlightingProportion(\%) & 8.3 & 9.1 & 10 & 11.1 & 12.5 & 14.3 & 16.7 & 20 & 25 & 33.3 & 50 & 100 & \\
\hline
\end{tabular}

TABLE 2: Observed mean and standard deviation of link travel times on 87 Route (unit: min).

\begin{tabular}{lcccccccccccccc}
\hline stop & 1 & 2 & 3 & 4 & 5 & 6 & 7 & 8 & 9 & 10 & 11 & 12 \\
\hline Mean & 1.46 & 2.05 & 0.89 & 1.87 & 1.66 & 1.65 & 1.63 & 4.41 & 0.82 & 0.79 & 0.83 & 1.35 & 0.2 \\
STD & 2 & 0.7 & 1.6 & 0.47 & 0.27 & 0.68 & 0.63 & 2.63 & 0.51 & 0.23 & 0.26 & 0.67 & 0.03 \\
Stop & 14 & 15 & 16 & 17 & 18 & 19 & 20 & 21 & 22 & 23 & 24 & 25 & 0.74 & 1.6 \\
Mean & 3.27 & 2.72 & 3.04 & 2.90 & 1.53 & 2.21 & 2.94 & 1 & 2.64 & 2.56 & 0.74 \\
STD & 1.07 & 0.75 & 1.22 & 1.36 & 0.48 & 1.03 & 1.06 & 0.28 & 0.73 & 0.66 & 0.27 & 0.28 & \\
\hline
\end{tabular}

Moreover, for a given slack ratio, we introduce two levels of schedule recovery effort: a low level uniformly distributed in an interval $[0.1,0.2]$ and a high level in an interval $[0.4,0.5]$. The results are shown in Figure 3. A summary of findings and their implications is listed as follows.

(a) In aspect of HVC, the $\mathrm{HH}$ control generally outperforms the $\mathrm{SH}$ under various slack ratios (Figure 3(a)). However, in the provision of schedule recovery, the $\mathrm{HH}$ is not always better than $\mathrm{SH}$ control in passenger waiting time $E(\bar{w})$, when the slack ratio lies between 0.6 and 1.2 (Figure $3(\mathrm{~b})$ ). The average travel time under $\mathrm{HH}$ control is shorter than that of $\mathrm{SH}$ when the slack ratio is larger than 2 (Figure 3(c)). When the slack ratio ranges from 0.6 to 1.2 , the $\mathrm{SH}$ with high level of schedule recovery presents the less variability and most uniform pattern in bus loads (Figure 3(d)).

(b) The load variation (Figure 3(d)) under $\mathrm{SH}$ first decreases and then increases with the increasing slack ratio. There are two possible reasons for this. First, 1.2 is already a high slack ratio to mitigate travel time variability; any improvement of headway stability could become more difficult through increasing the slacks. Second, higher slacks lead to less frequent service and greater accumulated boarding demand, which could in turn result in high crowding at some stops.

(c) Performance improvements for all indicators are observed at more sophisticated holding strategies with SR. The improvement is more significant when more schedule recovery effect is made. This is because schedule recovery compensates holding times vehicle spending. For SH control, schedule recovery could improve performance by a greater degree when the slack ratio is smaller. The reason is that when the slack time is sufficiently large, most of the travel time randomness and resulting delay have been mitigated, such that schedule recovery takes less effect. Therefore, one can see that when the slack ratio is relatively small, the benefit of HVC, waiting time, and travel time gained by schedule recovery is greater with $\mathrm{SH}$ control.

Naturally, a longer slack time for $\mathrm{SH}$ will lead to better schedule adherence, but at an expense of the negative effect of less frequent service and greater mean headways. As shown in Figure 3(b), such negative effect would overweight the reduction of headway variation when the slack time reaches a threshold (about 1); thereafter the waiting time increases instead. This suggests that sufficient holding times is not productive, and the operator should make a trade-off between the headway stability and efficiency in the planning. Hence, we analyse in the following the performance of holding control strategies with a reasonable level of slack ratio, at 1. Optimizing the slack time has been left for future study. In addition, to highlight the effect of schedule recovery effort, from now on the schedule recovery factor is set as a high level, i.e., uniformly distributed in an interval [0.4, 0.5]. The control policies are compared under the same operational settings, except where they are the subject of a test. This is approximated in the interest of presenting the incremental improvement, though it might be possible to improve performance further by optimizing headway.

4.2. Sensitivity to Travel Time Variability. In this section, we analyse the performance improvement from schedule recovery under various levels of travel time variability. The performance improvement is calculated as relative performances between with and without SR controls: $(S H-S H S R) \times$ $100 / S H S R$ or $(H H-H H S R) \times 100 / H H S R$. The travel time variability is reflected by the standard deviation of truncated normal distributed link travel time. We amplify the standard deviation by factors $1.2,1.4,1.6,1.8$, and 2 . The results are presented in Figure 4.

When the travel time variability increases, more performance improvement could be achieved by schedule recovery. While schedule recovery benefits both of the holding control strategies, the performance improvement of SHSR is much greater than that of HHSR under the same level of travel time variability. Since schedule recovery acts as the contributor to service recovery, these results suggest that $\mathrm{SH}$ is more sensitive to schedule recovery than $\mathrm{HH}$ in the presence of the travel time variability. In other words, SH method is less able to stabilize headways but could benefit more from schedule 


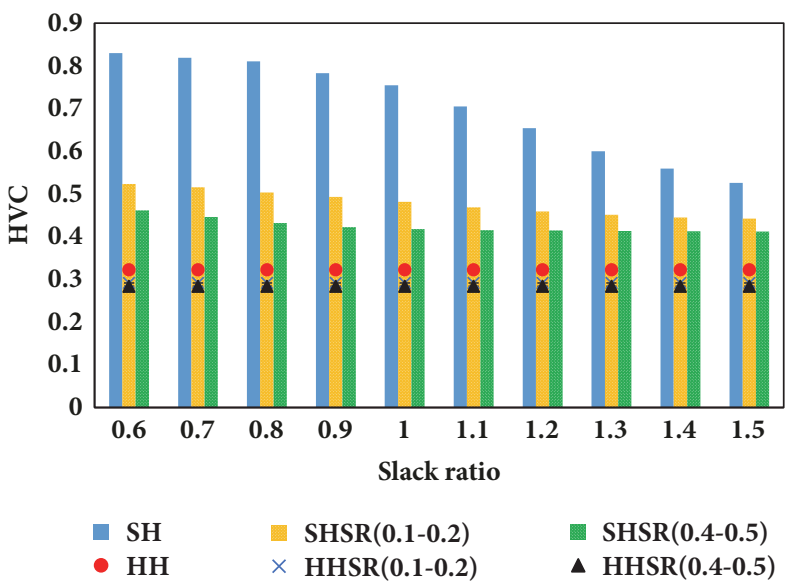

(a)

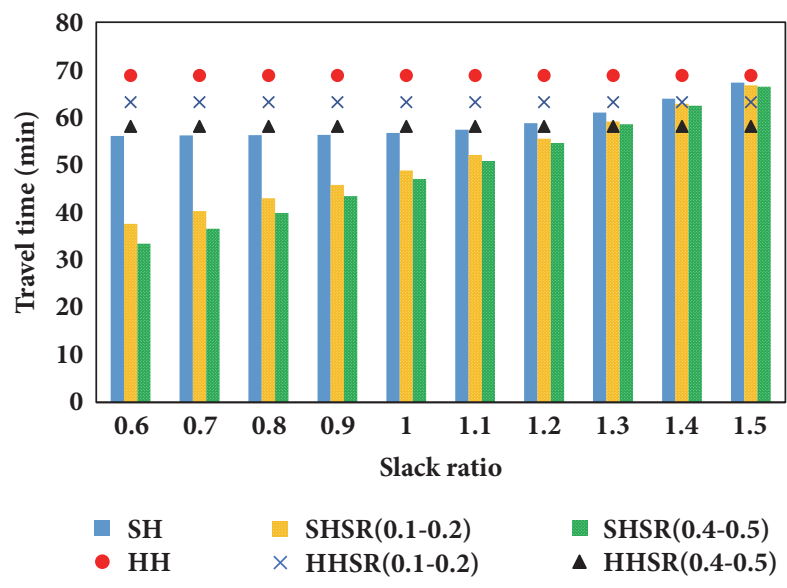

(c)

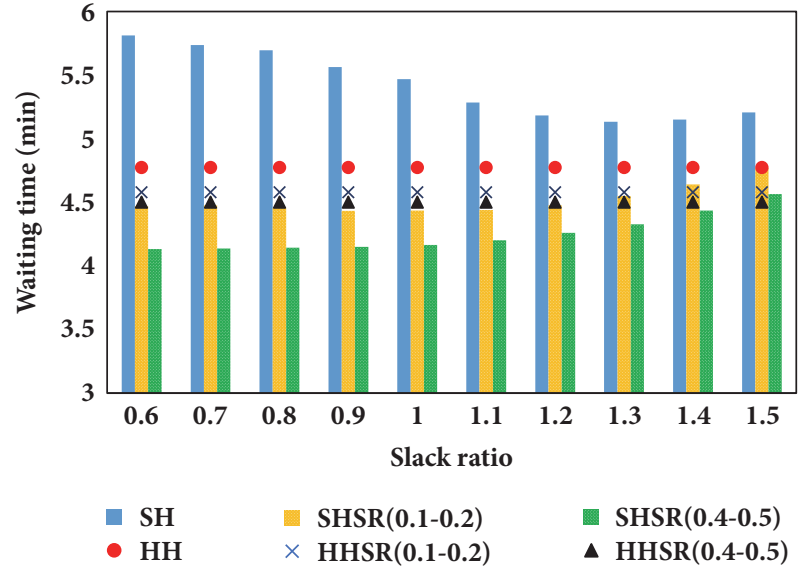

(b)

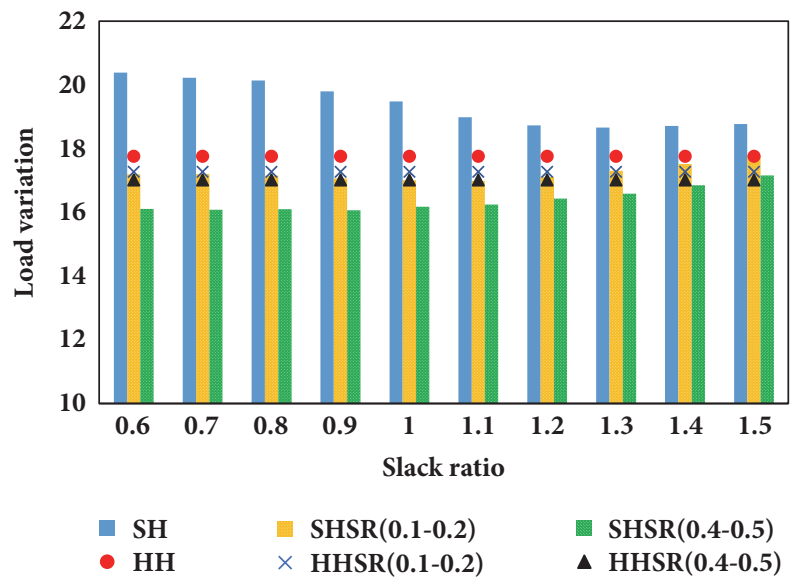

(d)

FIgURE 3: Performance measures under different control policies: (a) HVC; (b) $E(\bar{w})$; (c) average travel time; (d) load variation.

recovery. This implies that, in practice, cruising guidance technology has more effect in $\mathrm{SH}$ rather than $\mathrm{HH}$ under the same schedule recovery effort.

4.3. Sensitivity to Total Demand. Demand is one of the most important factors affecting the performance of the control strategy. To analyse the independent effect of demand congestion on the holding control performance, in this section we vary the demand levels without changing the headway and vehicle capacity. Figure 5 shows performance measures with varying demand levels.

SH appears to take no effect in terms of HVC in high demands (Figure 5(a)). A possible reason is that the effect of SH method is closely related to dwell times; when the demand volume grows (unless exceeding the capacity), buses are likely to stay longer at stops and depart later than the given scheduled departure time. Such effect may propagate along the downstream route. Consequently, the schedule departure times in $\mathrm{SH}$ takes less effect as the demand volume grows. This suggests that operators should pay attention to the level of passenger demand when using $\mathrm{SH}$ method.

$\mathrm{HH}$ is more sensitive to demand variation than $\mathrm{SH}$ in terms of average waiting time (Figure 5(b)). HH works well only in low demand; when the demand increases, the control performance deteriorates quickly in the average waiting time. In particular, $\mathrm{SH}$ outperforms $\mathrm{HH}$ in terms of the average waiting time when the demand ratio is larger than 2 . This is because, as discussed previously, $\mathrm{SH}$ takes less effect with higher demand, whereas $\mathrm{HH}$ is always in effect regardless of the demand level. In this sense, the service frequency under $\mathrm{HH}$ will be reduced as the demand increases as opposed to SH.

Bus load variation for both $\mathrm{SH}$ and $\mathrm{HH}$ increases with the demand ratio but at a decreasing rate (Figure 5(d)). This is because when the demand level reaches a threshold, the bus capacity can only meet the transport demand of the first several bus stops, after which buses tend to be full of passengers at the following stops. Schedule recovery takes more effect in smoothing bus loads under SH compared to $\mathrm{HH}$. As the demand ratio reaches 2 , the effect of schedule recovery is trivial for $\mathrm{HH}$.

4.4. Summary of the Key Findings and Their Practical Implications. In this section, we highlight the key findings from the sensitivity analysis of the proposed holding control with schedule recovery and discuss their operational implications. 


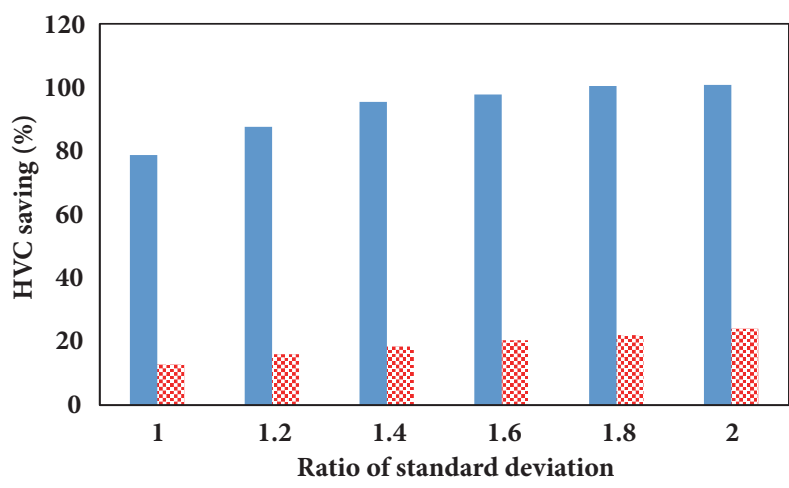

- $\mathrm{SH}$

$x$ HH

(a)

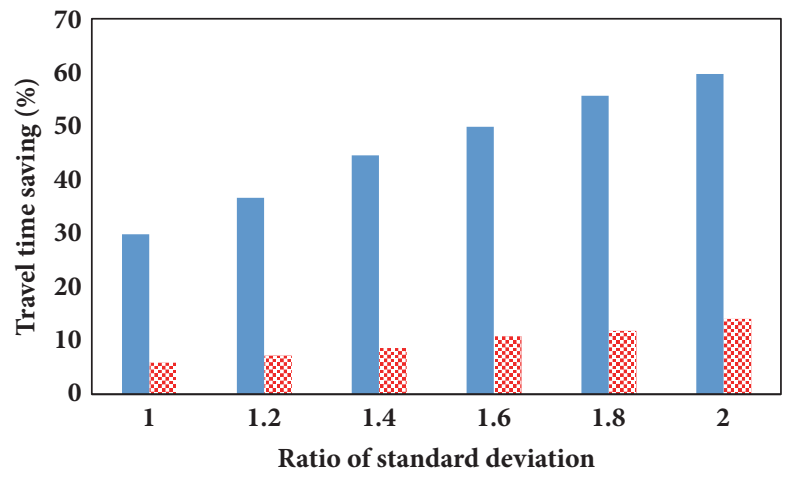

- $\mathrm{SH}$

$\therefore \mathrm{HH}$

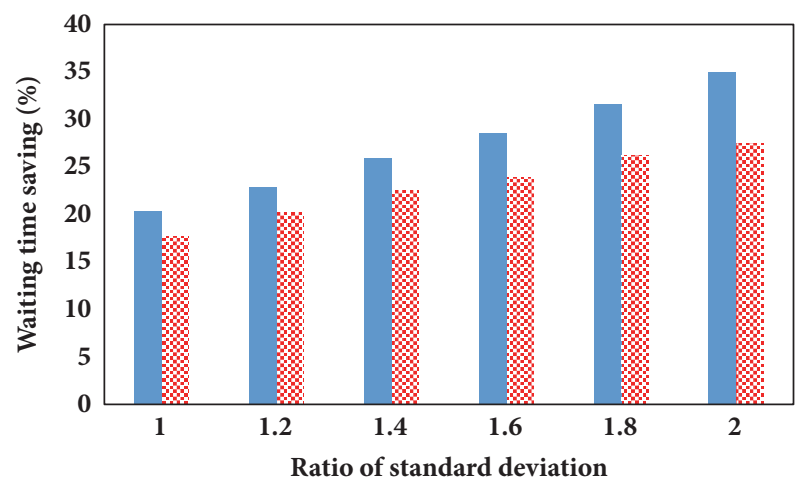

- $\mathrm{SH}$

\& $\mathrm{HH}$

(b)

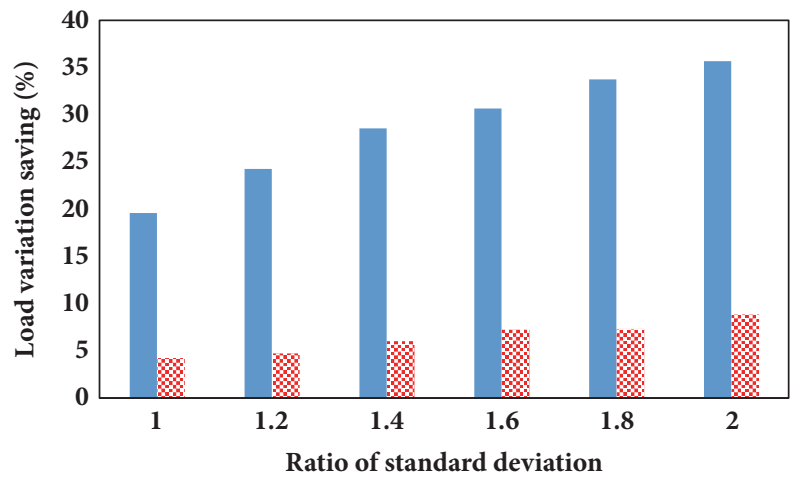

- $\mathrm{SH}$

* $\mathrm{HH}$

(c)

(d)

FIGURE 4: Percentage reductions in (a) HVC; (b) $E(\bar{w})$; (c) average travel time; (d) load variation under different travel time variation levels.

First, $\mathrm{HH}$ method generally outperforms the SH method with respect to HVC (Figure 3(a)). When schedule recovery (SR) effort is included; however, the relative performance of HHSR and SHSR would depend on the slack time. HHSR outperforms SHSR only with large slack ratios.

Second, we have shown that bus performance will always improve with SR effort, and SH control benefits more than $\mathrm{HH}$ control with SR, particularly in the presence of travel time variability (Figure 4). SR has less effect with larger slack ratios (Figure 3), and its effect in smoothing bus loads is greater under $\mathrm{SH}$ than under $\mathrm{HH}$ (Figure 5(d)).

Third, due to the inherent control mechanism, the SH takes less effect as the demand grows, whereas $\mathrm{HH}$ is always in effect. This may result in the phenomenon that SH outperforms $\mathrm{HH}$ in aspect of waiting time when the demand is sufficiently high (Figure 5(b)).

Based on the key findings described above, the following practical insights and recommendations can be drawn.

(a) Bus Stop Layout (Re)design. The above results show that cruising guidance and schedule recovery could improve the reliability of bus system. To facilitate the en route driver guidance and schedule recovery effort, some strategies can be introduced. For example, fewer stop activities and passenger flow control should be encouraged. According to the previous empirical analysis, bus stop consolidation has no significant effects on passenger activity [38]. Therefore, transit planners should make a trade-off between stop spacing and passengers' access to service in the design or redesign of bus route and possibly introduce wider stop spacing through the removal or consolidation of existing stops. Since the bus service time at bus stop areas usually occupies a large proportion of the total on-road bus operational time, it would also be helpful to invest in a quicker fare collection technology, such as building enclosed areas to allow off-bus fare collection for rapid boarding like the bus rapid transit system. In addition, for those bus routes traversing the center and suburb, such as Express Route 2 of Suzhou City in China, the stops in the suburban area with low demand could be converted into request stops (or flag stops), at which the vehicle will stop only on request.

(b) Determination of Slack Times. Including slack times in the schedule could stabilize headways, whereas too much slacks reduce service frequency. Transit operator need to consider the trade-off between the headway stability and efficiency in the planning. In the presence of schedule recovery, schedule-based method could achieve the best compromise 


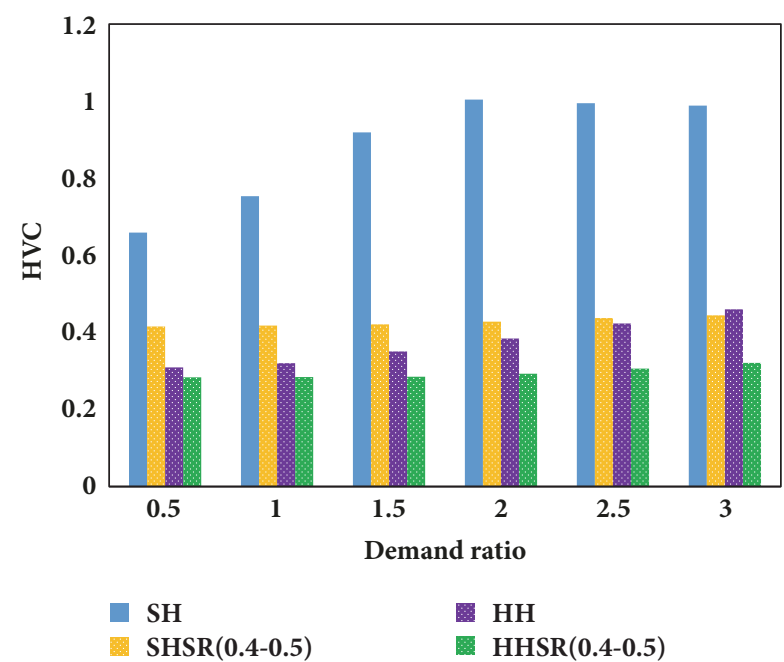

(a)

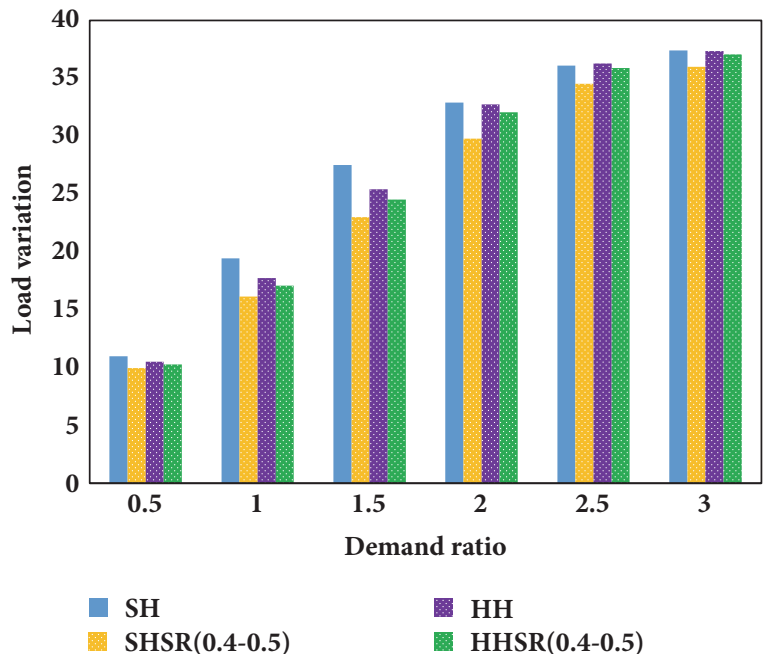

(c)

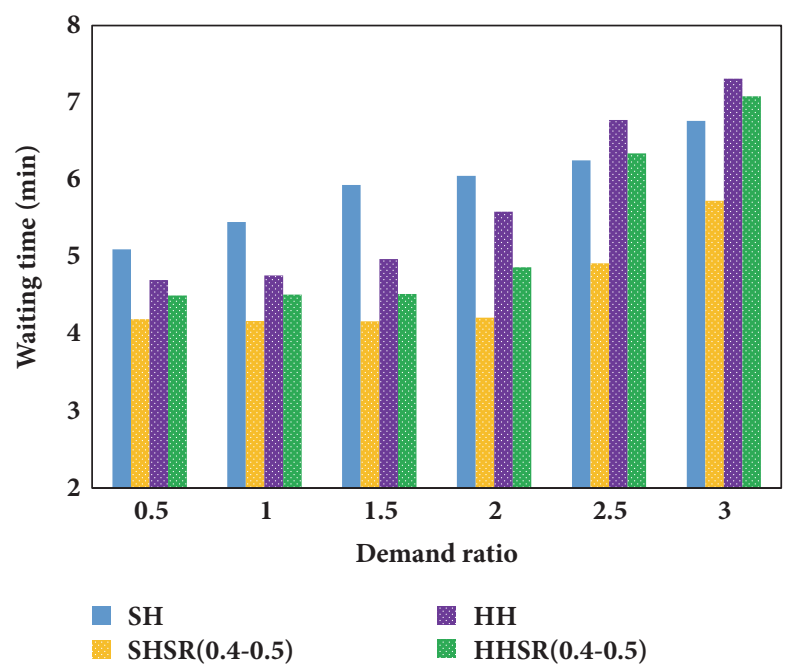

(b)

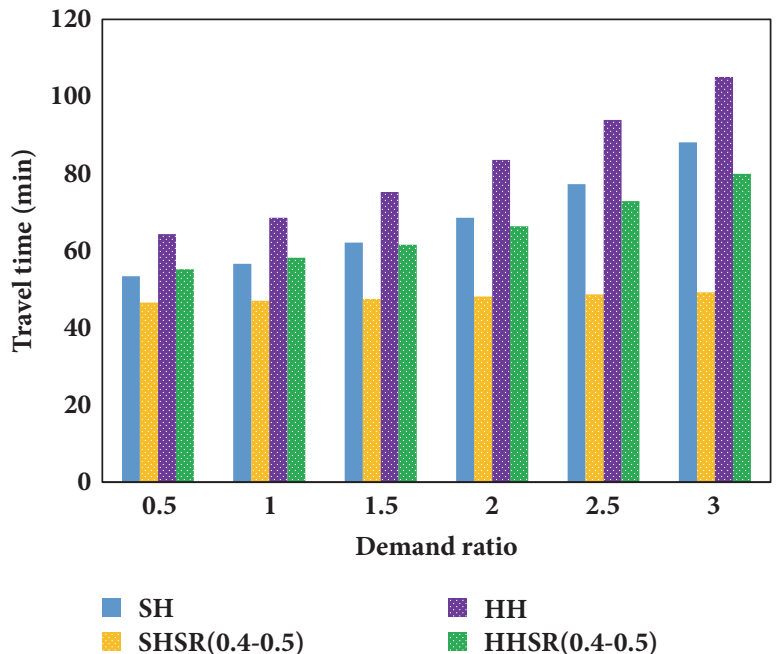

(d)

FIgURE 5: Performance measures for different total demand: (a) HVC; (b) $E(\bar{w})$; (c) average travel time; (d) load variation.

between headway regularity and efficiency with reasonable amounts of slack times, making it the preferable method for routes to reduce the waiting time.

(c) Selection of Bus Holding Control Method. Simulation results show that $\mathrm{SH}$ could benefit more from schedule recovery compared to $\mathrm{HH}$ under the same level of schedule recovery effort. Such property can be utilized to choose proper tactics in response to different operational conditions. Since there exist potential safety issues for schedule recovery, such as speeding and pushing traffic lights when there is little time left for the driver to catch up with the arrival schedule, the $\mathrm{SH}$ is preferable in the case of dedicated bus lanes, exclusive right of ways, or wide stop spacing where cruising guidance and schedule recovery could be more easily implemented. On the other hand, the $\mathrm{HH}$ may be recommended when the cruising guidance technology is absent or restricted, such as in normal lanes exposed to exogenous conditions. This is also the case during peak hours and traffic congestion, which implies that $\mathrm{HH}$ might be converted to $\mathrm{SH}$ in the transitional period from peak to off-peak hours, vice versus. Therefore, in practice, transit agencies should be mindful of choosing bus control methods in the context of cruising guidance technology. In addition, since SH will take less effect with higher demand as opposed to $\mathrm{HH}$, transit operators should pay attention to the level of passenger demand when choosing control method.

\section{Concluding Remarks}

This study was conducted to understand the impact of schedule recovery on real-time holding control strategies for a fixed bus route. Such schedule recovery behavior is readily available in the context of cruising guidance technology but so far has been neglected in the holding control analysis and evaluation. 
To capture the stochastic nature of travel times and demand, we first developed a bus propagation model with vehicle capacity constraint, which was extended by combining with the real-time holding control strategies (i.e., schedule-based and headway-based) and the effect of schedule recovery. The recovery time is proportional to the arriving delay time and the corresponding adjustment factor. Such stop-specific factor could be calibrated with the help of AVLAPC data.

The combined effects of holding control and schedule recovery were tested through a case study for a simulated real-life bus route in Guangzhou, China. We analysed and compared how the schedule recovery behavior affects the system performance of two holding control methods under different operating settings. We found that schedule recovery acts as the contributor to service reliability, and that schedulebased holding method is less able to stabilize headways but could benefit more from schedule recovery compared to headway-based holding method. The findings are specific to a real-life simulated bus route. The comparative results can help provide supporting tool for different bus control options and bus stop design in the provision of emerging technologies.

Future research may continue to develop an extended list of operational tactics and compare the combined effects under various operational settings. There exist common-line corridors where several bus lines serve the same stops [39]. The further study will also extend to considering multiple bus lines and investigate the impact of common-line stops on the system performance.

\section{Notations}

$i$ : The subscript of vehicle

$j: \quad$ The subscript of bus stop

C: The vehicle capacity

$q_{j}$ : The passenger arrival rate at stop $j$ (pax/min)

$\rho_{j}$ : The alighting proportion at stop $j(\%)$

$b$ : The average boarding time for passengers (s)

$\alpha$ : The average alighting time for passengers (s)

$H$ : The design headway of a bus line (min)

$\beta_{i, j}$ : The adjustment factor between stop $j$ and $j+1$ for bus $i$

$\delta: \quad$ Minimum safety interval (min)

$h_{i, j}$ : The headway between bus $i$ and the preceding bus at stop $j$ ( $\mathrm{min}$ )

$a_{i, j}$ : The arrival time of bus $i$ at stop $j$ (min)

$d_{i, j}$ : The departure time of bus $i$ at stop $j$ (min)

$t_{i, j}$ : The travel time between stop $j$ and $j+1$ for bus $i$ ( $\mathrm{min}$ )

$B_{i, j}$ : The total number of waiting passengers for bus $i$ at stop $j$ during the headway (pax)

$\bar{B}_{i, j}$ : The actual number of boarding passengers for bus $i$ at stop $j$ during the headway (pax)
$\bar{B}_{i, j}^{\prime}$ : The actual number of arriving passengers at stop $j$ who are able to board bus $i$ during the headway (pax)

$A_{i, j}$ : The number of alighting passengers of bus $i$ at stop $j(\operatorname{pax})$

$D_{i, j}:$ The dwell time for bus $i$ at stop $j$ (min)

$s_{i, j}$ : The scheduled departure time of bus $i$ at stop $j$ for schedule-based holding control (min)

$l_{i, j}$ : The number of leftover passengers of bus $i$ when it departs from stop $j$ (pax)

$L_{i, j}$ : The number of on-board passengers of bus $i$ between stop $j$ and $j+1$ (pax).

\section{Data Availability}

The data used to support the findings of this study are included within the article.

\section{Conflicts of Interest}

The authors declare that they have no conflicts of interest.

\section{Acknowledgments}

This work is supported by the National Science Foundation of China (Project nos. 61703165 and 61473122), the China Postdoctoral Science Foundation (Project no. 2016M600653), the Fundamental Research Funds for the Central Universities (Project no. D2171990), and the Royal Academy of Engineering (Project UK-CIAPP $\backslash 286$ ).

\section{References}

[1] Y. Hollander and R. Liu, "Estimation of the distribution of travel times by repeated simulation," Transportation Research Part C: Emerging Technologies, vol. 16, no. 2, pp. 212-231, 2008.

[2] W. Wu, R. Liu, and W. Jin, "Modelling bus bunching and holding control with vehicle overtaking and distributed passenger boarding behaviour," Transportation Research Part B: Methodological, vol. 104, pp. 175-197, 2017.

[3] E. E. Osuna and G. F. Newell, "Control strategies for an idealized public transportation system," Transportation Science, vol. 6, no. 1, pp. 52-72, 1972.

[4] M. D. Hickman, "An analytic stochastic model for the transit vehicle holding problem," Transportation Science, vol. 35, no. 3, pp. 215-237, 2001.

[5] X. J. Eberlein, N. H. M. Wilson, and D. Bernstein, "The holding problem with real-time information available," Transportation Science, vol. 35, no. 1, pp. 1-18, 2001.

[6] J. Zhao, M. Dessouky, and S. Bukkapatnam, "Optimal slack time for schedule-based transit operations," Transportation Science, vol. 40, no. 4, pp. 529-539, 2006.

[7] C. F. Daganzo, "A headway-based approach to eliminate bus bunching: Systematic analysis and comparisons," Transportation Research Part B: Methodological, vol. 43, no. 10, pp. 913-921, 2009.

[8] Y. Xuan, J. Argote, and C. F. Daganzo, "Dynamic bus holding strategies for schedule reliability: Optimal linear control and 
performance analysis," Transportation Research Part B: Methodological, vol. 45, no. 10, pp. 1831-1845, 2012.

[9] J. Argote-Cabanero, C. F. Daganzo, and J. W. Lynn, "Dynamic control of complex transit systems," Transportation Research Part B: Methodological, vol. 81, pp. 146-160, 2015.

[10] C. F. Daganzo and J. Pilachowski, "Reducing bunching with busto-bus cooperation," Transportation Research Part B: Methodological, vol. 45, no. 1, pp. 267-277, 2001.

[11] F. Delgado, J. C. Munoz, and R. Giesen, "How much can holding and/or limiting boarding improve transit performance?" Transportation Research Part B: Methodological, vol. 46, no. 9, pp. 1202-1217, 2012.

[12] D. Hernández, J. C. Muñoz, R. Giesen, and F. Delgado, "Analysis of real-time control strategies in a corridor with multiple bus services," Transportation Research Part B: Methodological, vol. 78, pp. 83-105, 2015.

[13] G. Sánchez-Martínez, H. N. Koutsopoulos, and N. H. M. Wilson, "Real-time holding control for high-frequency transit with dynamics," Transportation Research Part B: Methodological, vol. 83, pp. 1-19, 2016.

[14] B. Yu and Z. Yang, "A dynamic holding strategy in public transit systems with real-time information," Applied Intelligence, vol. 31, no. 1, pp. 69-80, 2009.

[15] L. Moreira-Matias, O. Cats, J. Gama, J. Mendes-Moreira, and J. F. De Sousa, "An online learning approach to eliminate Bus Bunching in real-time," Applied Soft Computing, vol. 47, pp. 460-482, 2016.

[16] R. Nair, E. Bouillet, Y. Gkoufas et al., "Data as a resource: realtime predictive analytics for bus bunching," in Proceedings of the Annual Meeting of the Transportation, Washington, D.C, 2014.

[17] M. Andres and R. Nair, "A predictive-control framework to address bus bunching," Transportation Research Part B: Methodological, vol. 104, pp. 123-148, 2017.

[18] A. Fonzone, J.-D. Schmöcker, and R. Liu, "A model of bus bunching under reliability-based passenger arrival patterns," Transportation Research Part C: Emerging Technologies, vol. 59, pp. 164-182, 2015.

[19] J.-D. Schmöcker, W. Sun, A. Fonzone, and R. Liu, "Bus bunching along a corridor served by two lines," Transportation Research Part B: Methodological, vol. 93, pp. 300-317, 2016.

[20] B. Yu, Z. Z. Yang, P. H. Jin, S. H. Wu, and B. Z. Yao, “Transit route network design-maximizing direct and transfer demand density," Transportation Research Part C: Emerging Technologies, vol. 22, pp. 58-75, 2012.

[21] W. Wu, R. Liu, and W. Jin, "Designing robust schedule coordination scheme for transit networks with safety control margins," Transportation Research Part B: Methodological, vol. 93, pp. 495519, 2016.

[22] M. Chen, X. Liu, and J. Xia, "Dynamic prediction method with schedule recovery impact for bus arrival time," Transportation Research Record, no. 1923, pp. 208-217, 2005.

[23] Y. Ji, L. He, and H. M. Zhang, "Bus drivers' responses to realtime schedule adherence and the effects on transit reliability," Transportation Research Record, vol. 2417, pp. 1-9, 2014.

[24] W.-H. Lin and R. L. Bertini, "Modeling schedule recovery processes in transit operations for bus arrival time prediction," Journal of Advanced Transportation, vol. 38, no. 3, pp. 347-365, 2004.

[25] Y. Yan, Q. Meng, S. Wang, and X. Guo, "Robust optimization model of schedule design for a fixed bus route," Transportation Research Part C: Emerging Technologies, vol. 25, pp. 113-121, 2012.
[26] T. Liu, A. Ceder, J. Ma, and W. Guan, "Synchronizing public transport transfers by using intervehicle communication scheme: Case study," Transportation Research Record, vol. 2417, pp. 78-91, 2014.

[27] M. M. Nesheli, A. Ceder, and V. A. Gonzalez, "RealTime Public-Transport Operational Tactics Using Synchronized Transfers to Eliminate Vehicle Bunching," IEEE Transactions on Intelligent Transportation Systems, vol. 17, no. 11, pp. 3220-3229, 2016.

[28] F. Milla, D. Sáez, C. E. Cortés, and A. Cipriano, "Bus-Stop Control Strategies Based on Fuzzy Rules for the Operation of a Public Transport System," IEEE Transactions on Intelligent Transportation Systems, 2012.

[29] J. B. Ingvardson, O. A. Nielsen, S. Raveau, and B. F. Nielsen, "Passenger arrival and waiting time distributions dependent on train service frequency and station characteristics: A smart card data analysis," Transportation Research Part C: Emerging Technologies, vol. 90, pp. 292-306, 2018.

[30] M. D. Salek and R. B. Machemehl, "Characterizing bus transit passenger waiting times," SWUTC Research Peport 99, Austin, Tex, USA, 1999.

[31] E. Hans, N. Chiabaut, L. Leclercq, and R. L. Bertini, "Real-time bus route state forecasting using particle filter and mesoscopic modeling," Transportation Research Part C: Emerging Technologies, vol. 61, pp. 121-140, 2015.

[32] Z. Liu, Y. Yan, X. Qu, and Y. Zhang, "Bus stop-skipping scheme with random travel time," Transportation Research Part C: Emerging Technologies, vol. 35, pp. 46-56, 2013.

[33] W. Wu, L. Shen, X. Ji, and W. Jin, "Analysis of freeway service patrol with discrete event-based simulation," Simulation Modelling Practice and Theory, vol. 47, pp. 141-151, 2014.

[34] B. Z. Yao, P. Hu, X. H. Lu, J. J. Gao, and M. H. Zhang, “Transit network design based on travel time reliability," Transportation Research Part C: Emerging Technologies, vol. 43, pp. 233-248, 2014.

[35] M. A. Turnquist and L. A. Bowman, "The effects of network structure on reliability of transit service," Transportation Research Part B: Methodological, vol. 14, no. 1-2, pp. 79-86, 1980.

[36] R. Liu and S. Sinha, Modelling urban bus service and passenger reliability. Paper presented at the International Symposium on Transportation Network Reliability, The Hague, Netherlands, 2007.

[37] W. Wu and W. Jin, "Comparative analysis of bus holding control strategies with the effect of schedule recovery," in Proceedings of the 97th Annual Meeting of the Transportation Research Board, Washington, DC, USA.

[38] A. M. El-Geneidy, J. G. Strathman, T. J. Kimpel, and D. T. Crout, "Effects of bus stop consolidation on passenger activity and transit operations," Transportation Research Record, no. 1971, pp. 32-41, 2006.

[39] B. Yu, W. H. K. Lam, and M. L. Tam, "Bus arrival time prediction at bus stop with multiple routes," Transportation Research Part C: Emerging Technologies, vol. 19, no. 6, pp. 1157-1170, 2011. 


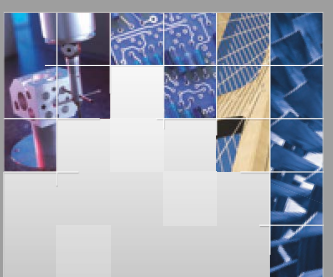

\section{Enfincering}
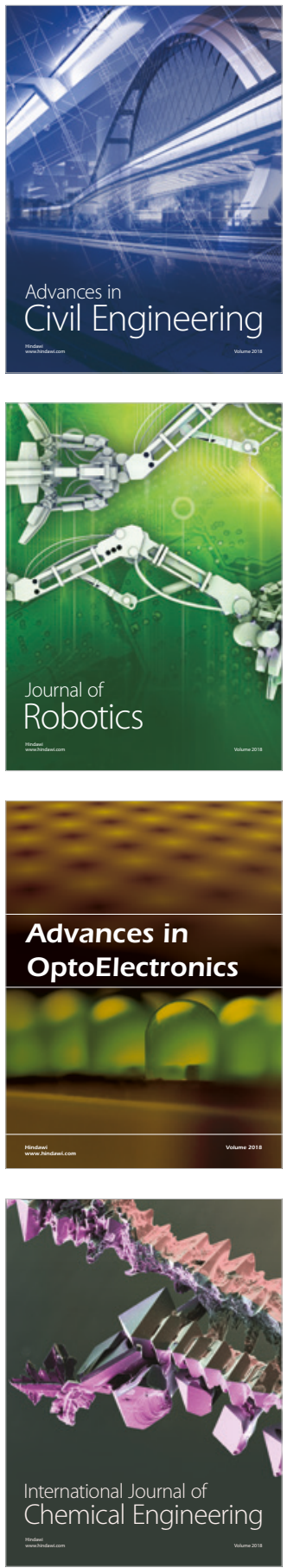

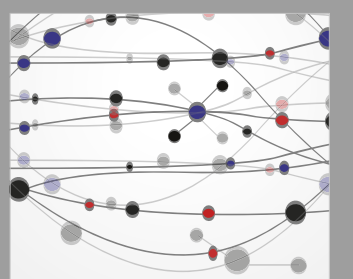

\section{Rotating \\ Machinery}

The Scientific World Journal

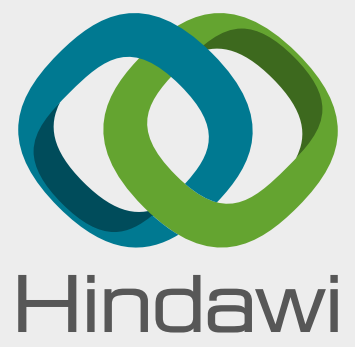

Submit your manuscripts at

www.hindawi.com
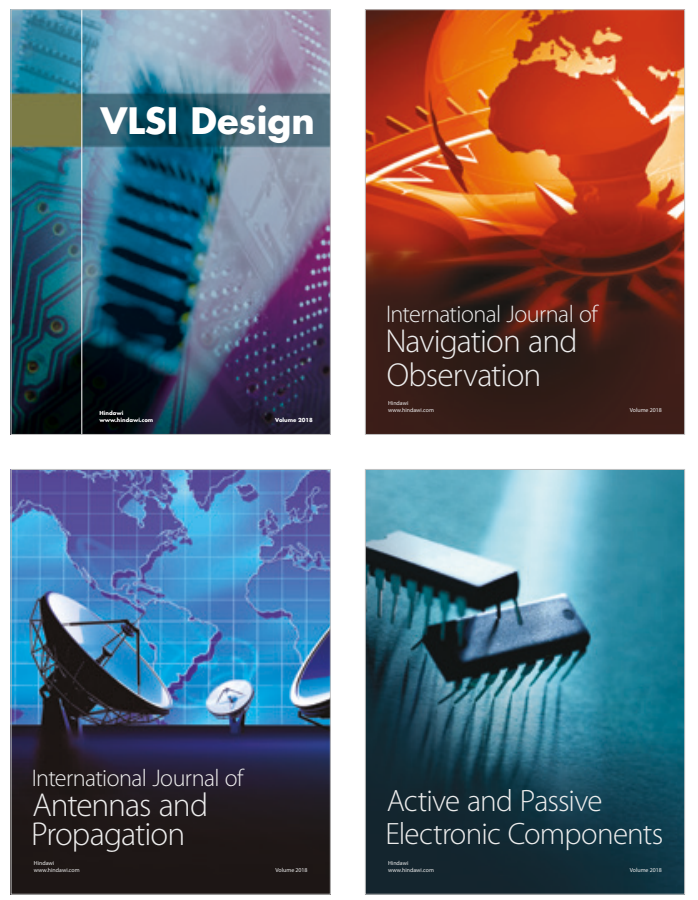
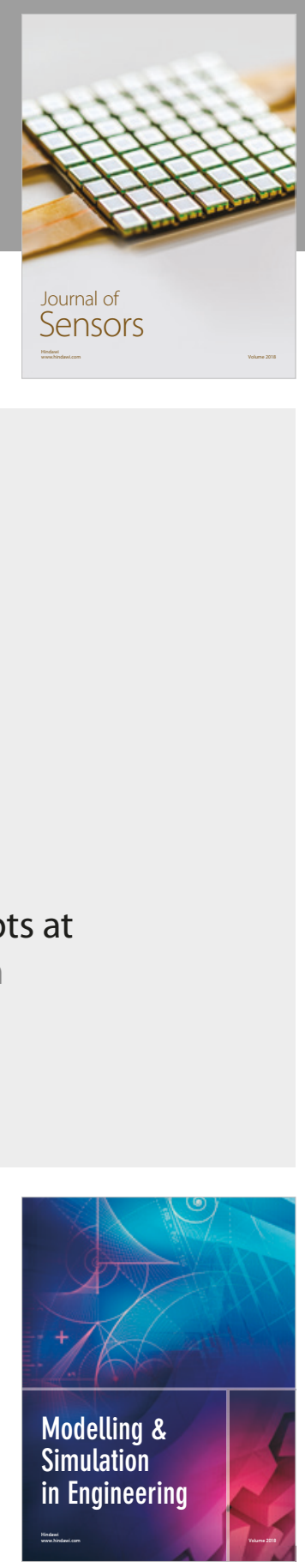

\section{Advances \\ Multimedia}
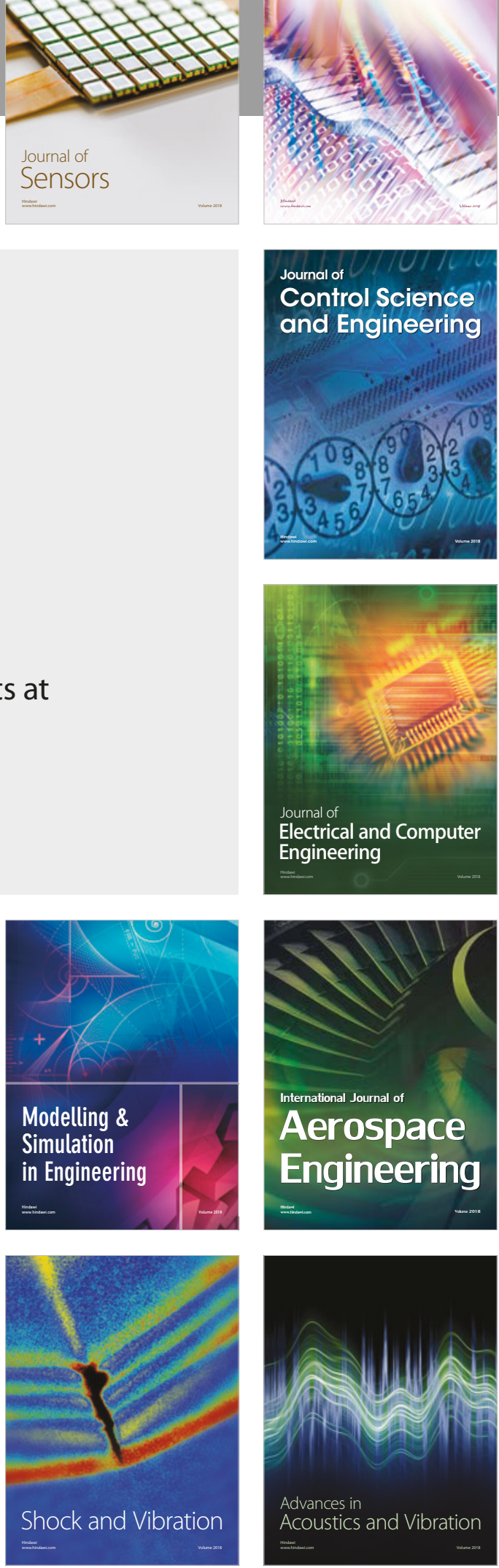